\title{
Clinicopathological implications of NQO1 overexpression in the prognosis of pancreatic adenocarcinoma
}

\author{
MEIYING JI ${ }^{1}$, AIHUA JIN ${ }^{2}$, JIE SUN ${ }^{1}$, XUELIAN CUI ${ }^{1}$, YANG YANG ${ }^{1}$, LIYAN CHEN ${ }^{1,3}$ and ZHENHUA LIN ${ }^{1}$ \\ ${ }^{1}$ Cancer Research Center and Department of Pathology, Yanbian University Medical College, \\ Yanji, Jilin 133002; ${ }^{2}$ Department of Internal Medicine, Yanbian University Hospital, Yanji, Jilin $133000 ;{ }^{3}$ Department \\ of Biological Chemistry and Molecular Biology, Yanbian University Medical College, Yanji, Jilin 133002, P.R. China
}

Received January 26, 2016; Accepted October 21, 2016

DOI: $10.3892 / \mathrm{ol} .2017 .5821$

\begin{abstract}
Nicotinamide adenine dinucleotide phosphate: quinone oxidoreductase 1 (NQO1) protects cells from oxidative damage. NQO1 has been reported to be upregulated in numerous solid tumors, suggesting a role in carcinogenesis and tumor progression. The present study attempted to investigate the clinical prognostic significance of NQO1 overexpression in pancreatic ductal adenocarcinoma (PDAC). A total of 181 tissue specimens were studied, including 126 PDAC and 55 normal pancreas specimens, which were selected for immunohistochemical staining of NQO1 protein. Immunofluorescence staining was additionally performed to identify the subcellular localization of NQO1 protein in pancreatic cancer BxPC-3 cells. The association between NQO1 overexpression and the clinical features of PDAC were evaluated by $\chi^{2}$ and Fisher's exact test. Overall survival of PDAC patients was calculated using Kaplan-Meier analysis. Univariate and multivariate analyses were performed using the Cox proportional hazards regression model. The NQO1 protein was mainly located in the cytoplasm and nucleus of BxPC-3 cells. The strongly positive rate of NQO1 expression in PDAC $(65.9 \%, 83 / 126)$ was increased compared with that in normal pancreatic tissues $(10.9 \%, 6 / 55)$. The positive rate of NQO1 protein was associated with grading, lymph node stage and tumor-node-metastasis (TNM) stage. Additionally, multivariate analysis suggested that NQO1 was a significant independent prognostic factor along with TNM stage in PDAC. In conclusion, high expression of NQO1 appears to be associated with PDAC progression, and may be an independent prognostic biomarker in PDAC.
\end{abstract}

Correspondence to: Professor Liyan Chen or
Professor Zhenhua Lin, Cancer Research Center and
Department of Pathology, Yanbian University Medical College,
977 Gongyuan Road, Yanji, Jilin 133002, P.R. China
E-mail: lychen@ybu.edu.cn
E-mail: zhlin720@ybu.edu.cn

Key words: pancreatic ductal adenocarcinoma, NQO1, immunohistochemistry, prognosis, survival analysis

\section{Introduction}

Pancreatic ductal adenocarcinoma (PDAC) is one of the most common types of malignant tumors of the digestive tract, and it has the worst prognosis of all major malignancies, with a 5 -year survival rate of $6 \%$ and a median survival of 6 months subsequent to diagnosis $(1,2)$. Although numerous advances in prevention, surgical resection and adjuvant chemoradiotherapy have led to a decline in the overall mortality due to PDAC, the survival rates for patients with metastatic disease have not significantly improved $(3,4)$. Therefore, the identification of an ideal marker for early diagnosis and prognosis evaluation of PDAC has been an important research field in clinical examination.

Nicotinamide adenine dinucleotide phosphate (NADPH): quinone oxidoreducase 1 (NQO1) is located on chromosome 16q22, and consists of six exons and five introns (5). NQO1 is a flavin adenine dinucleotide-dependent direct two-electron reductase that can use NADH or NADPH as reducing cofactors to reduce quinones to hydroquinones (6). Several functions of NQO1 have been reported, including xenobiotic detoxification, superoxide scavenging, modulation of p53, maintenance of endogenous antioxidants and proteasomal degradation (7), suggesting that NQO1 is important in protecting normal cells against oxidative injury and carcinogenesis (8). NQO1 protein was also observed to be abnormally expressed in numerous human tumors $(9,10)$. However, there is limited research investigating the associations between NQO1 expression and PDAC development and progression.

Thus, the current study aimed to demonstrate the association between NQO1 expression and clinicopathological characteristics of patients with PDAC. The present results revealed that NQO1 was significantly upregulated in PDAC, which was associated with tumor grade, tumor node-metastasis (TNM) stage, lymph node (LN) metastasis and overall survival (OS) of patients with PDAC. NQO1 may be an independent prognostic biomarker for patients with PDAC.

\section{Materials and methods}

Ethics statement. The present study complied with the principles of the Declaration of Helsinki, and was approved by the human ethics and research ethics committees of Yanbian 
University Medical College (Yanji, China). Patients provided written informed consent and were informed that resected specimens were stored by the hospital, and potentially used for scientific research, and their privacy would be maintained. Follow-up survival data were collected retrospectively through medical record analyses.

Clinical specimens. A total of 181 tissue specimens were studied, including 126 PDAC tissues and 55 normal pancreas specimens. All tissues were obtained from Shanghai Outdo Biotech Co., Ltd. (Shanghai, China) and the Tissue Bank of Yanbian University Medical College. All tissues were fixed in $10 \%$ buffered formalin and paraffin embedded. The present study protocol was approved by the institutional review board of Yanbian University Medical College. Pathological parameters consisted of age, gender, tumor size, differentiation, pathological grading, TNM stage, LN metastasis and survival data. In total, 74 patients were $>50$ years old and 52 patients were below 50 years of age. TNM staging was assessed according to the staging system established by the American Joint Committee on Cancer (AJCC) (11-13). The male-to-female ratio was 71:55. Staging was performed according to the TNM stage, and 72 patients were stage I-II while 54 patients were stage III-IV. In addition, the ratio of tumor grading was 36(G1):37(G2):53(G3). Among the 126 patients with PDAC, 74 patients had LN metastasis while 52 patients did not have $\mathrm{LN}$ metastasis. In the 126 patients with PDAC, the follow-up time was 30-67 months.

Immunofluorescence (IF) staining for NQO1 protein in BxPC-3 cells. BxPC-3 was purchased from the Korean Cell Line Bank (Seoul, Korea). The cells were grown on glass coverslips in Dulbecco's modified Eagle's medium (Gibco; Thermo Fisher Scientific, Inc., Waltham, MA, USA) media with $10 \%$ heat inactivated fetal bovine serum (Gibco; Thermo Fisher Scientific, Inc.). The cells were incubated at $37^{\circ} \mathrm{C}$ in an atmosphere containing $5 \%$ $\mathrm{CO}_{2}$ and at $95 \%$ humidity, to between 70 and $80 \%$ confluence. The cells were fixed $4 \%$ paraformaldehyde for $20 \mathrm{~min}$ at room temperature (RT) and permeabilized with $0.1 \%$ Triton X-100 for $10 \mathrm{~min}$. The cells were then washed with PBS, and 5\% bovine serum albumin was next added for $30 \mathrm{~min}$ at RT. The cells were subsequently incubated with anti-NQO1 antibody (dilution, 1:200; cat. no. A180:sc-32793; Santa Cruz Biotechnology, Inc., Dallas, TX, USA) at $4^{\circ} \mathrm{C}$ overnight. The cells were then incubated with Alexa Fluor ${ }^{\circledR} 568$ Goat Anti-Mouse IgG (H+L) secondary antibody (dilution, 1:200; cat. no. A11004; Invitrogen; Thermo Fisher Scientific, Inc.) for $1 \mathrm{~h}$ at RT. Subsequent to washing with PBS, cells were counterstained with DAPI (cat. no. C1006; Beyotime Institute of Biotechnology, Shanghai, China), and the coverslips were mounted with Antifade Mounting Medium (cat. no. P0126; Beyotime Institute of Biotechnology). Finally, the IF signals were visualized and recorded with a TCS SP5 II confocal microscope (Leica Microsystems, Inc., Buffalo Grove, IL, USA).

Immunohistochemical (IHC) analysis. IHC analysis was performed using the LSAB kit (Dako; Agilent Technologies, Inc., Santa Clara, CA, USA). Prior to IHC staining, all sections were deparaffinized, rehydrated and incubated with $3 \% \mathrm{H}_{2} \mathrm{O}_{2}$ in methanol for $15 \mathrm{~min}$ at RT. The antigen was retrieved at $95^{\circ} \mathrm{C}$ for $20 \mathrm{~min}$ by placing the slides in $0.01 \mathrm{M}$ sodium citrate buffer ( $\mathrm{pH}$ 6.0). The slides were then incubated with the aforementioned anti-NQO1 monoclonal antibody (dilution, 1:200) at $4^{\circ} \mathrm{C}$ overnight. Subsequent to incubation with a biotinylated secondary antibody (pre-diluted; cat. no. PV9000; OriGene Technologies, Inc., Beijing, China) at RT for $30 \mathrm{~min}$, the slides were incubated with a streptavidin-peroxidase complex (cat. no. PV9000; OriGene Technologies, Inc.) at RT for $30 \mathrm{~min}$. IHC staining was developed using 3,3'-diaminobenzidine, and Mayer's hematoxylin (cat. no.,ZLI9610; OriGene Technologies, Inc.) was used for counterstaining. Mouse immunoglobulin $\mathrm{G}$ (pre-diluted; cat. no. PV9000; OriGene Technologies, Inc.) was used as an isotype control, incubation at RT for $30 \mathrm{~min}$. A negative control was utilized by processing the tissue sections without the primary antibody.

Evaluation of IHC staining. All tissue specimens were blindly examined by two pathologists. In case of discrepancies, a final score was established by reassessment on a double-headed microscope. Immunostaining for NQO1 was semi-quantitatively scored as:,$-<5 \%$ positive cells;,$+ 5-25 \%$ positive cells;,$++ 26-50 \%$ positive cells; and,$+++>50 \%$ positive cells. Cytoplasmic or nuclear NQO1 staining was considered to be positive staining. Tissue sections scored as ++ , and +++ were considered to be strong positive staining. For survival data analysis, ++ or +++ scored samples were considered high-level NQO1 expression, while - or + scored samples were considered to exhibit low levels of NQO1 expression.

Statistical analysis. All statistical analyses were performed using SPSS (version 17.0; SPSS, Inc., Chicago, IL, USA) software. $\chi^{2}$ test and Fisher's exact test, were used to assess the association between clinicopathological characteristics and the expression of studied protein. The survival rates following tumor removal were calculated using the Kaplan-Meier estimator and differences in survival curves were analyzed using log-rank tests. Multivariate survival analysis was performed on all the significant characteristics measured by univariate survival analysis through the Cox proportional hazards regression model. $\mathrm{P}<0.05$ was considered to indicate a statistically significant difference.

\section{Results}

High expression of $N Q O 1$ protein in PDACs. The positive rate of NQO1 protein expression in PDAC tissue was significantly increased compared with that in normal pancreatic tissues $(83.3 \%, 105 / 126, \mathrm{P}<0.01)$. Similarly, the strongly positive rate of NQO1 expression in PDAC tissue was increased compared with that in normal pancreatic tissues $(65.9 \%, 83 / 126, \mathrm{P}<0.01$; Table I). IF staining revealed that NQO1 protein was mainly located in the cytoplasm and nucleus of BxPC-3 cells (Fig. 1). Consistently, IHC staining demonstrated that NQO1 protein localized to the cytoplasm of PDAC tissues (Fig. 2).

Clinicopathological significance of NQO1 expression in patients with PDAC. The present study investigated the association between NQO1 expression and the clinicopathological features of patients with PDAC. According to the clinical parameters listed in Table II, it was shown that NQO1 
Table I. Nicotinamide adenine dinucleotide phosphate:quinone oxidoreductase 1 protein expression in pancreatic ductal adenocarcinoma.

\begin{tabular}{lccccccc}
\hline & & \multicolumn{4}{c}{ Positive patients } & & Positive patient \\
\cline { 3 - 5 } Diagnosis & Patients, $\mathrm{n}$ & - & + & ++ & +++ & $\begin{array}{c}\text { Strongly positive } \\
\text { rates, } \%\end{array}$ \\
\hline PDAC tissue & 126 & 21 & 22 & 45 & 38 & $83.3^{\mathrm{a}}$ & $65.9^{\mathrm{a}}$ \\
Normal pancreas & 55 & 36 & 13 & 6 & 0 & 34.5 & 10.9 \\
\hline
\end{tabular}

${ }^{\mathrm{a}} \mathrm{P}<0.01$ compared with the normal pancreatic tissues.

overexpression was not associated with patient's age $(\mathrm{P}=0.153)$, gender $(\mathrm{P}=0.931)$, tumor size $(\mathrm{P}=0.192)$ or differentiation $(\mathrm{P}=0.050)$. However, it was observed that the positive rates of NQO1 expression in grade $2(\mathrm{G} 2 ; 70.3 \%, 26 / 37)$ and grade 3 (G3; 75.5\%, 40/53) were significantly increased compared with those in grade $1(\mathrm{G} 1 ; 47.2 \%, 17 / 36, \mathrm{P}=0.018)$. Additionally, NQO1 expression was higher in TNM stage III-IV (77.8\%, 42/54) compared with that in stage I-II $(56.9 \%, 41 / 72, \mathrm{P}=0.015)$. In addition, NQO1 expression was higher in PDAC patients with LN metastasis $(75.7 \%, 56 / 74)$ than in patients without LN metastasis (51.9\%, 27/52; P<0.01; Table II and Fig. 3).

To additionally confirm the importance of NQO1 in PDAC, the present study analyzed the OS of 162 patients with PDAC using the Kaplan-Meier method. Patients with high NQO1 expression exhibited a lower rate of OS compared with those with low NQO1 expression (log-rank=22.755, $\mathrm{P}<0.001$; Fig. 4A). Similarly, survival of patients with G1 (log-rank=7.317, $\mathrm{P}<0.01), \mathrm{G} 2(\log -\mathrm{rank}=8.025, \mathrm{P}<0.01)$ and $\mathrm{G} 3(\log -\mathrm{rank}=9.037$, $\mathrm{P}=0.003$ ) was significantly lower in PDAC tissues exhibiting high NQO1 expression compared with those that in tissues exhibiting low NQO1 expression (Fig. 4B-D). Patients with PDAC and NQO1 positive expression had lower OS rates compared with those without NQO1 expression in the absence (log-rank=19.291, $\mathrm{P}<0.001)$ and presence of $\mathrm{LN}$ metastasis (log-rank=5.259, $\mathrm{P}=0.022$ ) (Fig. 5). Furthermore, patients with PDAC exhibiting high NQO1 expression displayed decreased OS compared with those with low NQO1 expression in early stage patients (log-rank=18.402, $\mathrm{P}<0.001$; Fig. 6).

NQO1 expression is an independent prognostic biomarker in $P D A C$. Univariate analysis demonstrated that PDAC patients with NQO1 positive expression exhibited significant lower OS rate [hazard ratio $(\mathrm{HR})=1.715,95 \%$ confidence interval $(\mathrm{CI})=1.802-4.091, \mathrm{P}<0.001)$ compared with those with NQO1 negative expression. Additionally, tumor size $(\mathrm{HR}=1.444$, 95\% $\mathrm{CI}=1.008-2.068, \mathrm{P}=0.045)$, TNM stage $(\mathrm{HR}=2.148$, 95\% $\mathrm{CI}=1.487-3.102, \mathrm{P}<0.001)$ and $\mathrm{LN}$ metastasis $(\mathrm{HR}=1.466$, $95 \% \mathrm{CI}=1.028-2.091, \mathrm{P}=0.035)$ were all significantly associated with OS rates of PDAC patients. Furthermore, multivariate analysis was performed using the Cox proportional hazards model for all the variables examined in the univariate analysis. It was observed that NQO1 expression emerged as a significant independent prognostic factor for OS rates in patients with PDAC (HR=2.340, 95\% CI=1.409-3.884, $\mathrm{P}=0.001)$ along with TNM stage $(\mathrm{HR}=1.962,95 \% \mathrm{CI}=1.334-2.817, \mathrm{P}=0.002$; Table III).
Table II. Association between high expression of NQO1 protein and clinicopathological parameters of patients with pancreatic ductal adenocarcinoma.

\begin{tabular}{|c|c|c|c|}
\hline Variables & $\begin{array}{l}\text { Patients, } \\
\text { n }\end{array}$ & $\begin{array}{l}\text { NQO1 strongly } \\
\text { positive patients, } \\
\text { n (\%) }\end{array}$ & P-value \\
\hline \multicolumn{4}{|l|}{ Age, years } \\
\hline$\geq 50$ & 74 & $45(60.8)$ & \multirow[t]{2}{*}{0.153} \\
\hline$<50$ & 52 & $38(73.1)$ & \\
\hline \multicolumn{4}{|l|}{ Gender } \\
\hline Male & 71 & $47(66.2)$ & \multirow[t]{2}{*}{0.931} \\
\hline Female & 55 & $36(65.4)$ & \\
\hline \multicolumn{4}{|c|}{ Tumor size, cm } \\
\hline$<3$ & 75 & $46(61.3)$ & \multirow[t]{2}{*}{0.192} \\
\hline$\geq 3$ & 51 & $37(72.5)$ & \\
\hline \multicolumn{4}{|c|}{ Differentiation } \\
\hline Well & 37 & $21(56.8)$ & \multirow[t]{3}{*}{0.050} \\
\hline Moderately & 42 & $26(61.9)$ & \\
\hline Poorly & 47 & $36(76.6)$ & \\
\hline \multicolumn{4}{|l|}{ Grading } \\
\hline G1 & 36 & $17(47.2)$ & \multirow[t]{3}{*}{0.018} \\
\hline $\mathrm{G} 2$ & 37 & $26(70.3)$ & \\
\hline G3 & 53 & $40(75.5)$ & \\
\hline \multicolumn{4}{|l|}{ TNM stage } \\
\hline I-II & 72 & $41(56.9)$ & \multirow[t]{2}{*}{0.015} \\
\hline III-IV & 54 & $42(77.8)$ & \\
\hline \multicolumn{4}{|l|}{ LN metastasis } \\
\hline Absence & 52 & 27 (51.9) & \multirow[t]{2}{*}{0.006} \\
\hline Presence & 74 & $56(75.7)$ & \\
\hline
\end{tabular}

NQO1, nicotinamide adenine dinucleotide phosphate:quinone oxidoreductase 1; TNM, tumor-node-metastasis; LN, lymph node.

\section{Discussion}

NQO1 was identified by Ernster and Navazio in 1958 (14) as a cytosolic flavoenzyme that reduces quinones to less toxic hydroquinones in a single two-electron transfer step (15). Subsequent to decades of research, it is currently known that 
Table III. Univariate and multivariate analyses of clinicopathological factors for the overall survival rate of 126 patients with pancreatic ductal adenocarcinoma.

\begin{tabular}{|c|c|c|c|c|}
\hline \multirow[b]{2}{*}{ Characteristics } & \multicolumn{2}{|c|}{ Univariate analysis } & \multicolumn{2}{|c|}{ Multivariate analysis } \\
\hline & $\mathrm{HR}(95 \% \mathrm{CI})$ & P-value & HR $(95 \%$ CI) & P-value \\
\hline Age & $1.241(0.864-1.782)$ & 0.242 & $1.306(0.876-1.947)$ & 0.189 \\
\hline Gender & $1.346(0.935-1.938)$ & 0.110 & $0.799(0.526-1.215)$ & 0.295 \\
\hline Tumor size & $1.444(1.008-2.068)$ & 0.045 & $1.294(0.890-1.880)$ & 0.177 \\
\hline Differentiation & $1.086(0.878-1.343)$ & 0.446 & $1.067(0.842-1.353)$ & 0.592 \\
\hline Grading & $1.094(0.887-1.350)$ & 0.399 & $0.856(0.678-1.081)$ & 0.192 \\
\hline TNM stage & $2.148(1.487-3.102)$ & 0.000 & $1.962(1.334-2.817)$ & 0.002 \\
\hline LN metastasis & $1.466(1.028-2.091)$ & 0.035 & $1.441(0.986-2.106)$ & 0.059 \\
\hline NQO1 expression & $2.715(1.802-4.091)$ & 0.000 & $2.340(1.409-3.884)$ & 0.001 \\
\hline
\end{tabular}

HR, hazard ratio; CI, confidence interval; TNM, tumor-node-metastasis; LN, lymph node; NQO1, nicotinamide adenine dinucleotide phosphate: quinone oxidoreductase 1 .
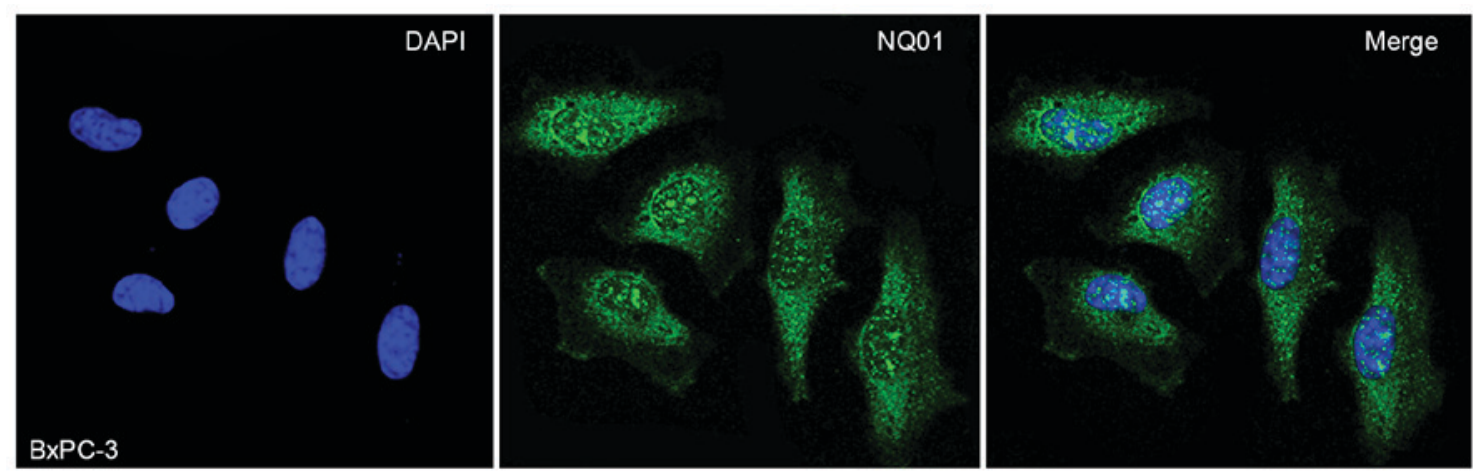

Figure 1. Immunofluorescent staining of NQO1 protein in BxPc-3 cells. NQO1 protein located in the cytoplasm and nucleus of BxPc-3 cells (green indicates NQO1; blue indicates DAPI; original magnification, x630). NQO1, nicotinamide adenine dinucleotide phosphate:quinone oxidoreductase 1.

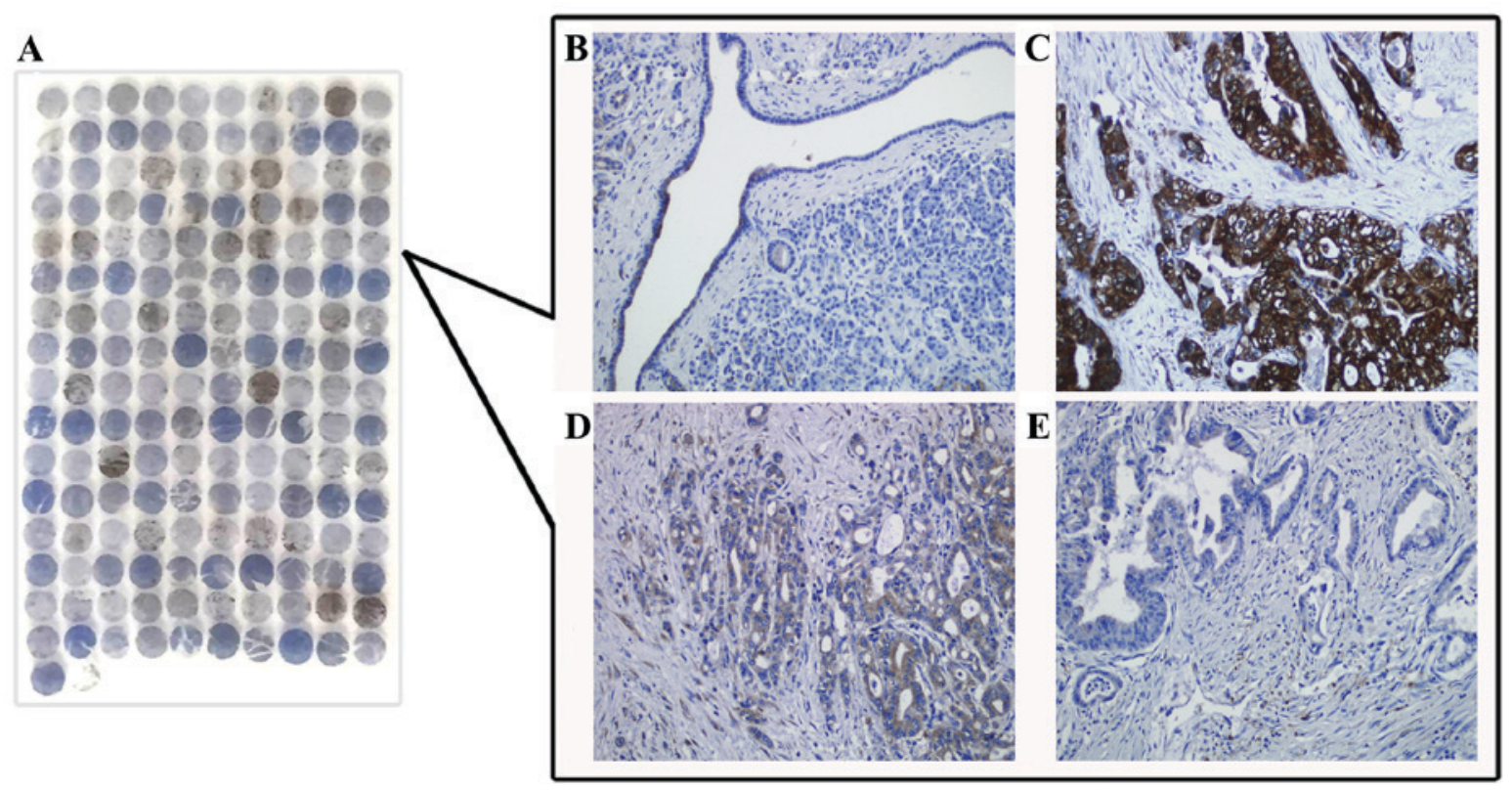

Figure 2. Immunohistochemical staining of NQO1 protein expression in PDAC tissues. (A) NQO1 protein expression was evaluated in microarray of PDAC tissues. (B) NQO1 staining demonstrated negative expression in non-tumor tissues. (C) Strongly positive expression of NQO1 protein in PDAC with metastasis. (D) NQO1 protein was weakly positive expressed in PDAC. (E) NQO1 protein was negatively expressed in PDAC (Original magnification, x200 in B-E). NQO1, nicotinamide adenine dinucleotide phosphate:quinone oxidoreductase 1; PDAC, pancreatic ductal adenocarcinoma. 

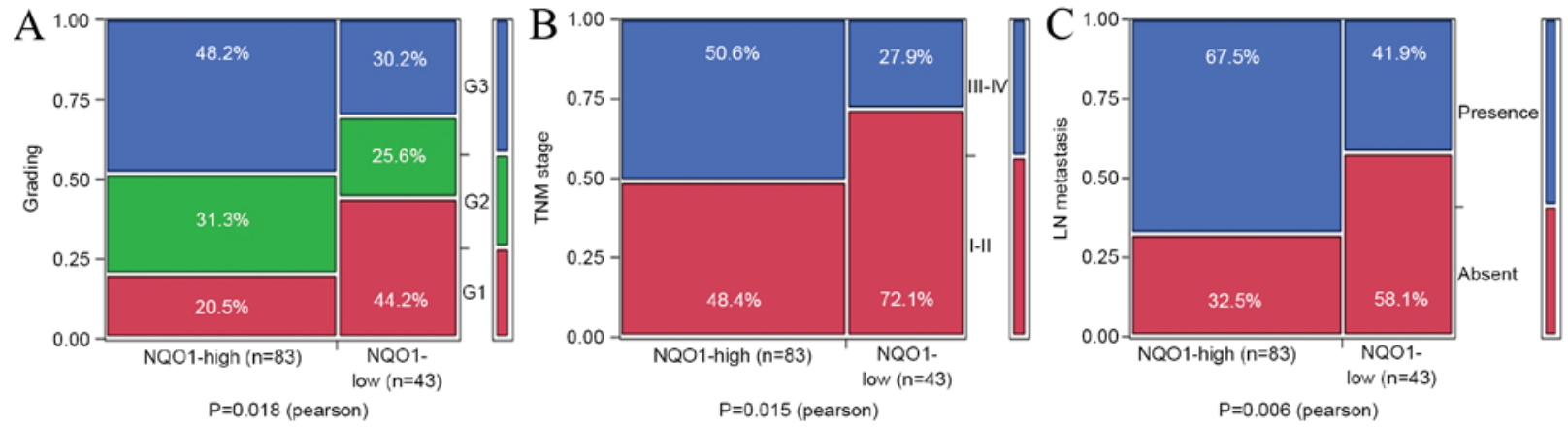

Figure 3. Association between NQO1 expression and clinicopathological significance of PDAC. The expression level of NQO1 protein was significantly associated with $(\mathrm{A})$ grading $(\mathrm{P}<0.05)$, (B) TNM stage $(\mathrm{P}<0.05)$ and $(\mathrm{C}) \mathrm{LN}$ metastasis $(\mathrm{P}<0.01)$. NQO1, nicotinamide adenine dinucleotide phosphate:quinone oxidoreductase 1; PDAC, pancreatic ductal adenocarcinoma; TMN, tumor-node-metastasis; LN, lymph node.

A

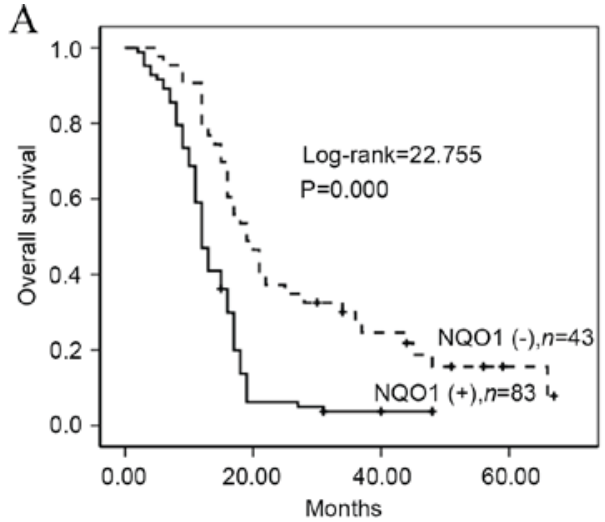

$\mathrm{C}$

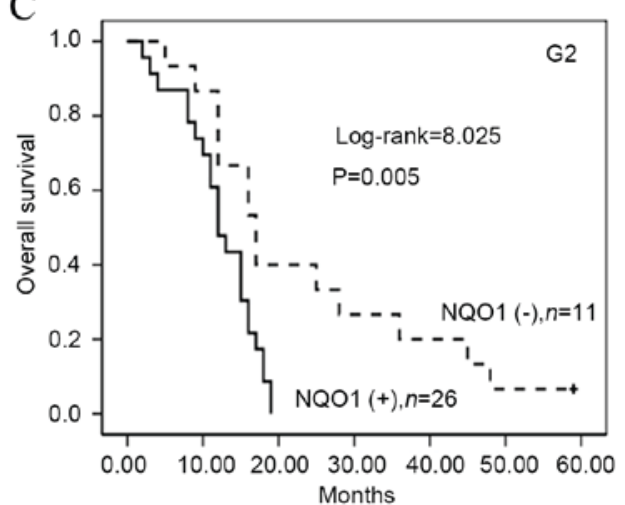

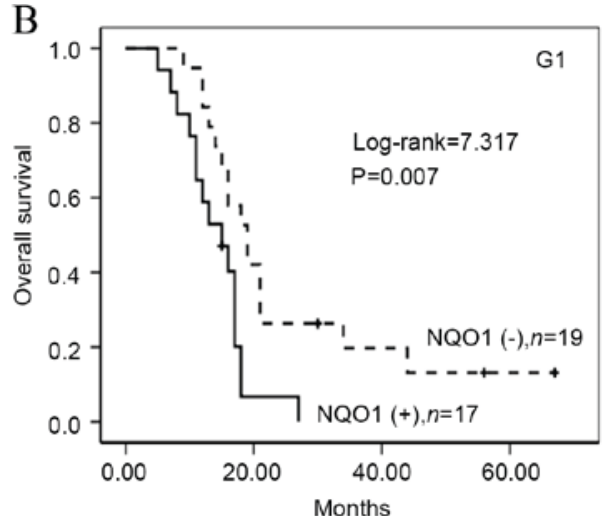

$\mathrm{D}$

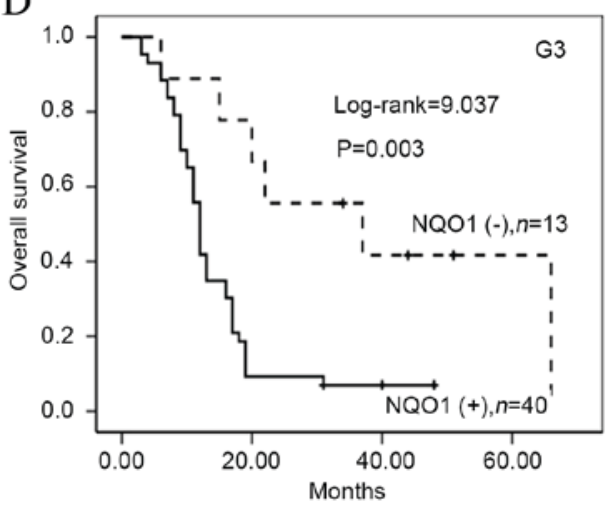

Figure 4. Kaplan-Meier survival curves illustrating the significance of NQO1 expression in PDAC patients with different grading. (A) OS rates of patients with high (solid; $\mathrm{n}=83$ ) and low (dashed; $\mathrm{n}=43$ ) NQO1 expression $(\mathrm{P}<0.001)$. High NQO1 expression was strongly associated with poor OS in (B) G1 $(\mathrm{P}=0.007)$, (C) G2 ( $\mathrm{P}=0.005)$ and (D) G3 ( $\mathrm{P}=0.003)$. NQO1, nicotinamide adenine dinucleotide phosphate:quinone oxidoreductase 1; PDAC, pancreatic ductal adenocarcinoma; OS, overall survival.

NQO1 can induce a series of defensive factors to prevent the damage caused by exogenous substances, oxidants, and ultraviolet and ionizing radiation (16). NQO1 is additionally involved in the maintenance of the active forms of coenzyme $\mathrm{Q}$ and vitamin $\mathrm{E}$, the regulation of the immune response and autoimmunity and the stabilization of the tumor suppressors p53, p73a and p33 (17).

As a cell protector, there is evidence that $\mathrm{NQO1}$ expression was significantly increased in the cytoplasm and nucleus of certain human solid tumors (18). Malkinson et al (19) observed that NQO1 was highly expressed in human lung cancer tissues. Cheng et al (20) demonstrated that NQO1 expression was significantly increased in primary melanomas compared with that in dysplastic nevi, and that this may occur in the initiation stage of melanoma development. Our previous studies have shown that the expression of NQO1 protein was significantly high in non-small cell lung cancer (21), serous ovarian carcinoma (22) and gastric adenocarcinoma (23), indicating that the expression of NQO1 was associated with the occurrence of tumors, and that it may be a significant prognostic or predictive marker. However, the role of NQO1 as a biomarker in PDAC is unclear.

In the present study, IHC staining of NQO1 protein in PDAC tissue was performed, and it was observed that staining 
A

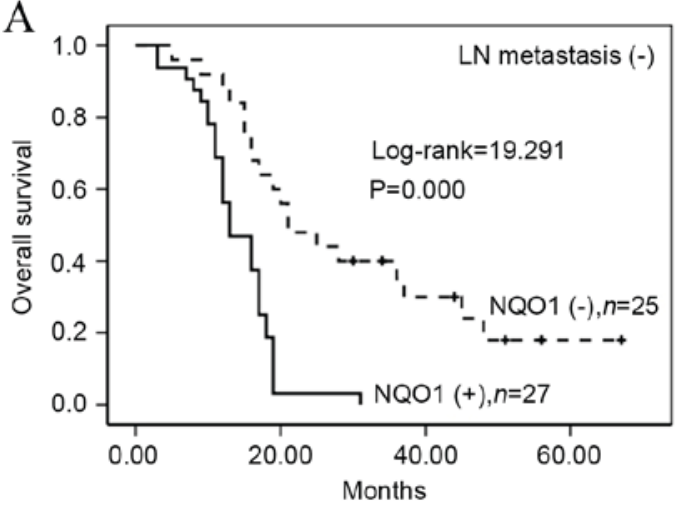

B

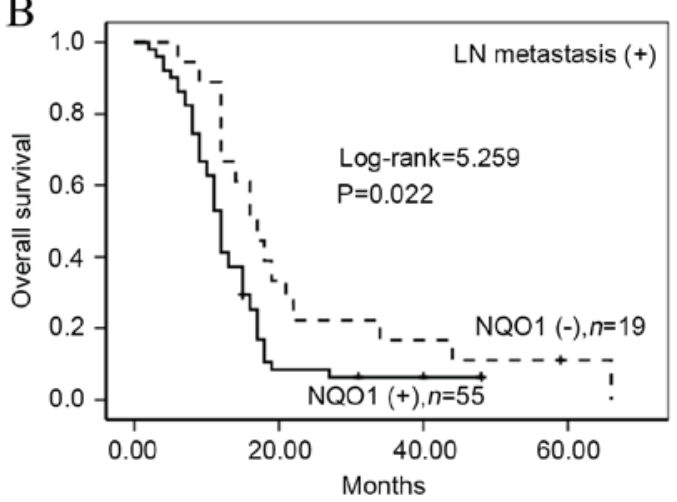

Figure 5. Kaplan-Meier survival curves of PDAC patients with lymph node metastasis and without metastasis. (A) Kaplan-Meier curves for OS in the absence of LN metastasis in PDAC patients with low and high level of NQO1 expression (log-rank=18.402, P<0.001). (B) Kaplan-Meier curves for OS in the presence of LN metastasis in PDAC patients with low level and high level of NQO1 expression (log-rank=5.259, P=0.022). PDAC, pancreatic ductal adenocarcinoma; OS, overall survival; NQO1, nicotinamide adenine dinucleotide phosphate:quinone oxidoreductase 1; LN, lymph node.

A

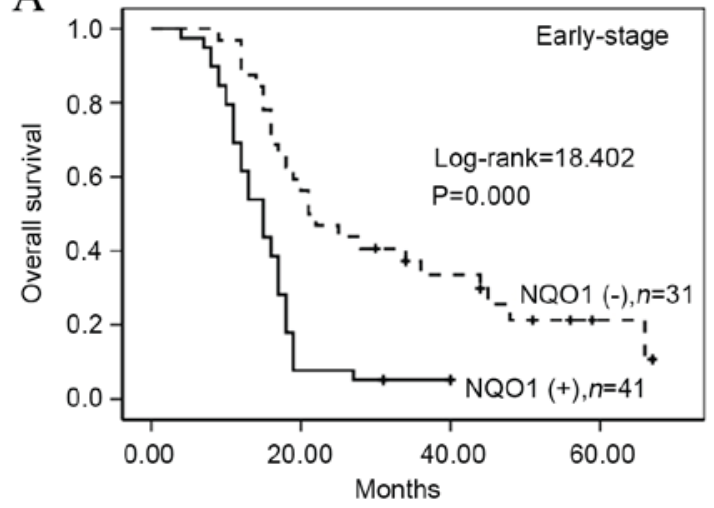

B

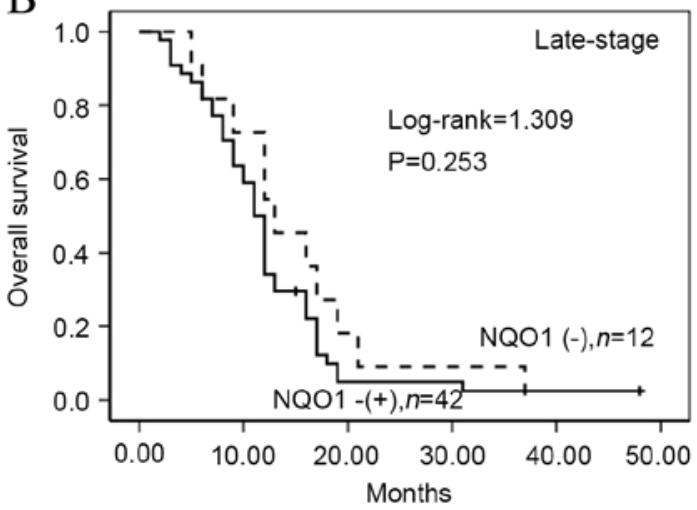

Figure 6. Kaplan-Meier survival curves of PDAC patients in early and late stage. (A) Kaplan-Meier curves for OS in early-stage PDAC patients with low and high level of NQO1 expression (log-rank=18.402, $\mathrm{P}<0.001$ ). (B) Kaplan-Meier curves for OS in late-stage PDAC patients with low and high level of NQO1 expression (log-rank=1.309, $\mathrm{P}=0.253$ ). PDAC, pancreatic ductal adenocarcinoma; OS, overall survival; NQO1, nicotinamide adenine dinucleotide phosphate: quinone oxidoreductase 1 ; LN, lymph node.

of NQO1 is localized in the cytoplasm. IF staining also revealed that NQO1 protein localized in the cytoplasm and nucleus of BxPC-3 cells. In these tissues, the strongly positive rate of NQO1 protein was 65.9\% (83/126) in PDAC tissue, which was significantly greater than that in normal pancreas tissue, indicating that NQO1 may serve an important role in the progression of PDAC. The current study analyzed the association between high expression of NQO1 protein and the clinicopathological parameters of PDAC; the results indicated that the rate of strongly positive NQO1 protein expression in G2 and G3 PDAC was increased compared with that in G1 PDAC. Additionally, high NQO1 expression was significantly associated with LN metastasis and TNM stage, which is consistent with the study by Mikami et al (24), suggesting that NQO1 upregulation promotes the invasion and metastasis of PDAC.

Buranrat et al (25) reported a significant association between high level of NQO1 expression and short OS time of patients with cholangiocarcinoma, which raised the possibility of using NQO1 as a tumor marker. In the present study, univariate survival analysis revealed that tumor size $(\mathrm{P}=0.045)$, TNM stage $(\mathrm{P}<0.001)$, LN metastasis $(\mathrm{P}=0.035)$ and NQO1 expression $(\mathrm{P}<0.001)$ status were all significantly associated with OS of patients with serous PDAC. Additionally, multivariate survival analysis revealed that NQO1 expression was a prognostic factor of PDAC, along with TNM stage. Overall, the present results indicate that NQO1 may be a biomarker for early diagnosis and prognosis, and a potential molecular target in patients with PDAC.

Recent studies have reported that anti-tumor agents such as $\beta$-lapachone and deoxynyboquinone (DNQ) effectively kill cancer cells through NQO1 specific activation $(26,27)$. Huang et al reported that the potency and NQO1-dependent therapeutic window of DNQ and its apparent reduced metabolism by one-electron oxidoreductases make DNQ or its derivatives a promising avenue for study (28).

In conclusion, NQO1 is important in the occurrence of PDAC, and may serve as an important marker and potential therapeutic target for PDAC. Therefore, additional research may verify whether NQO1 inhibitors could be used in the treatment of patients with PDAC.

\section{Acknowledgements}

The present study was supported by grants from the National Natural Science Foundation of China (grant nos. 81460399 and 61371067). 


\section{References}

1. Siegel R, Naishadham D and Jemal A: Cancer statistics, 2013 CA Cancer J Clin 63: 11-30, 2013.

2. Michl P and Gress TM: Current concepts and novel targets in advanced pancreatic cancer. Gut 62: 317-326, 2013.

3. Charpentier M and Martin S: Interplay of stem cell characteristics, EMT and microtentacles in circulating breast tumor cells. Cancers (Basel) 5: 1545-1565, 2013.

4. Cuppone F, Bria E, Vaccaro V, Puglisi F, Fabi A, Sperduti I, Carlini P, Milella M, Nisticò C, Russillo M, et al: Magnitude of risks and benefits of the addition of bevacizumab to chemotherapy for advanced breast cancer patients: Meta-regression analysis of randomized trials. J Exp Clin Cancer Res 30: 54, 2011.

5. Rosvold EA, McGlynn KA, Lustbader ED and Buetow KH: Re: Detection of a point mutation in NQO1 (DT-diaphorase) in a patient with colon cancer. J Natl Cancer Inst 87: 1802-1803, 1995.

6. Schlager JJ and Powis G: Cytosolic NAD(P)H (Quinone-acceptor)oxidoreductase in human normal and tumor tissue: Effects of cigarette smoking and alcohol. Int J Cancer 45: 403-409, 1990.

7. Buranrat B, Prawan A, Kukongviriyapan U, Kongpetch S and Kukongviriyapan V: Dicoumarol enhances gemcitabine-induced cytotoxicity in high NQO1-expressing cholangiocarcinoma cells. World J Gastroenterol 16: 2362-2370, 2010.

8. Su XL, Yan MR and Yang L; Qimuge-Suyila: NQO1 C609T polymorphism correlated to colon cancer risk in farmers from western region of Inner Mongolia. Chin J Cancer Res 24: 317-322, 2012.

9. Siegel D, Franklin WA and Ross D: Immunohistochemical detection of $\mathrm{NAD}(\mathrm{P}) \mathrm{H}$ : Quinone oxidoreductase in human lung and lung tumors. Clin Cancer Res 4: 2065-2070, 1998.

10. Siegel D and Ross D: Immunodetection of NAD(P)H: Quinone oxidoreductase 1 (NQO1) in human tissues. Free Radic Biol Med 29: 246-253, 2000

11. Sobin L, Gospodarowicz M and Wittekind C: TNM Classification of Malignant Tumours. 7th edition. Wiley Blackwell, Bognor Regis, UK, 2009.

12. Edge S, Byrd DR, Compton CC, Fritz AG, Greene FL and Trotti A: AJCC cancer staging manual. Springer, New York, NY, 2010.

13. Bosman F, Carneiro F, Hruban RH and Theise ND: Who classification of Tumours of the Digesitive System. IARC Press, Lyon, 2010.

14. Ernster L and Navazio F: Soluble diaphorase in animal tissues. Acta Chem Scand 12: 595-602, 1958.

15. Ross D, Kepa JK, Winski SL, Beall HD, Anwar A and Siegel D: NAD(P)H: Quinone oxidoreductase 1 (NQO1): Chemoprotection, bioactivation, gene regulation and genetic polymorphisms. Chem Biol Interact 129: 77-97, 2000.
16. Garate M, Wani AA and Li G: The NAD(P)H: Quinone oxidoreductase 1 induces cell cycle progression and proliferation of melanoma cells. Free Radic Biol Med 48: 1601-1609, 2010.

17. Iskander K, Li J, Han S, Zheng B and Jaiswal AK: NQO1 and NQO2 regulation of humoral immunity and autoimmunity. J Biol Chem 281: 30917-30924, 2006.

18. Siegel D, Kepa JK and Ross D: NAD(P)H: Quinone oxidoreductase 1 (NQO1) localizes to the mitotic spindle in human cells. PLoS One 7: e44861, 2012.

19. Malkinson AM, Siegel D, Forrest GL, Gazdar AF, Oie HK, Chan DC, Bunn PA, Mabry M, Dykes DJ, Harrison SD, et al: Elevated DT-diaphorase activity and messenger RNA content in human non-small cell lung carcinoma: Relationship to the response of lung tumor xenografts to mitomycin $\mathrm{Cl}$. Cancer Res 52: 4752-4757, 1992.

20. Cheng Y, Li J, Martinka M and Li G: The expression of NAD(P)H: Quinone oxidoreductase 1 is increased along with NF-kB p105/p50 in human cutaneous melanomas. Oncol Rep 23: 973-979, 2010.

21. Li Z, Zhang Y, Jin T, Men J, Lin Z, Qi P, Piao Y and Yan G: NQO1 protein expression predicts poor prognosis of non-small cell lung cancers. BMC Cancer 15: 207, 2015.

22. Cui X, Li L, Yan G, Meng K, Lin Z, Nan Y, Jin G and Li C: High expression of NQO1 is associated with poor prognosis in serous ovarian carcinoma. BMC cancer 15: 244, 2015.

23. Lin L, Qin Y, Jin T, Liu S, Zhang S, Shen X and Lin Z: Significance of NQO1 overexpression for prognostic evaluation of gastric adenocarcinoma. Exp Mol Pathol 96: 200-205, 2014.

24. Mikami K, Naito M, Tomida A, Yamada M, Sirakusa T and Tsuruo T: DT-diaphorase as a critical determinant of sensitivity to mitomycin $\mathrm{C}$ in human colon and gastric carcinoma cell lines. Cancer Res 56: 2823-2826, 1996.

25. Buranrat B, Chau-In S, Prawan A, Puapairoj A, Zeekpudsa P and Kukongviriyapan V: NQO1 expression correlates with cholangiocarcinoma prognosis. Asian Pac J Cancer Prev 13 (Suppl): S131-S136, 2012.

26. Kuang HN, Weng TY, Liu YL, Lu KS and Chau YP: Sulidac compounds facilitate the cytotoxicity of $\beta$-lapachone by up-regulation of $\mathrm{NAD}(\mathrm{P}) \mathrm{H}$ quinone oxidoreductase in human lung cancer cells. PLoS One 9: e88122, 2014

27. Park EJ, Min KJ, Lee TJ, Yoo YH, Kim YS and Kwon TK: $\beta$-lapachone induces programmed necrosis through the RIP1-PARP-AIF-dependent pathway in human hepatocellular carcinoma SK-Kep1 cells. Cell Death Dis 5: e1230, 2014.

28. Huang X, Dong Y, Bey EA, Kilgore JA, Bair JS, Li LS, Patel M, Parkinson EI, Wang Y, Williams NS, et al: An NQO1 substrate with potent antitumor activity that selectively kills by PARP1-induced programmed necrosis. Cancer Res 72: 3038-3047, 2012. 